\title{
Suivi de la gravidité chez la brebis Ouled Djellal par dosage de la protéine associée à la gestation et de la progestérone
}

\author{
A. Benyounes ${ }^{1}$ F. Lamrani ${ }^{2}$ N. Melo de Sousa ${ }^{3}$ \\ J. Sulon ${ }^{3}$ J. Folch ${ }^{4}$ J.-F. Beckers ${ }^{3 *}$ M.A. Guellati $5 \S$
}

\begin{abstract}
Mots-clés
Ovin - Brebis Ouled Djellal Gestation - Progestérone - Technique radioimmunologique - Algérie.
\end{abstract}

\begin{abstract}
Résumé
L'objectif de la présente étude a été la caractérisation des profils plasmatiques de la protéine associée à la gestation (PAG) et de la progestérone, au cours de la gravidité et deux semaines après le part chez la race Ouled Djellal. Vingtcinq brebis ont été prélevées hebdomadairement après synchronisation des chaleurs et monte naturelle. Les concentrations de PAG et de progestérone ont été déterminées par dosage radio-immunologique. Deux femelles n'ont pas été fécondées tandis que 23 ont eu des gestations considérées comme normales $(n=18)$ ou pathologiques $(n=5)$. Chez 18 brebis (12 gestations simples et 6 multiples), les concentrations de PAG et de progestérone ont été significativement influencées par le stade de la gestation $(P<0,0001)$ et non par le nombre de fœetus. Des profils individuels ont été décrits chez sept brebis, dont trois femelles ayant eu des pertes embryonnaires, et deux brebis ayant avorté $\left(66^{\mathrm{e}}\right.$ et $110^{\mathrm{e}}$ jours), et enfin chez deux brebis ayant donné naissance à terme chacune à un agneau vivant et expulsé au moment de la mise bas un ou deux fœetus momifiés. Les concentrations en PAG ont été plus faibles chez les femelles ayant eu des fœetus momifiés en parallèle avec des fœetus vivants.
\end{abstract}

\section{INTRODUCTION}

La progestérone, première hormone stéroïde connue, joue un rôle indispensable dans le maintien de la gestation chez les mammifères euthériens (3). Chez la brebis, le placenta synthétise des quantités non négligeables de progestérone à partir du cinquantième jour après fécondation, assurant le maintien de la gravidité même s'il y a ovariectomie $(17,18)$. Le rôle indispensable de cette hormone chez les femelles gravides a été à la base du développement des premières méthodes de diagnostic hormonal de la gravidité dès les années 1970 (5).

1. Institut d'Agronomie, El Tarf, Algérie.

2. Institut technique des Elevages, Alger, Algérie.

3. Faculté de Médecine vétérinaire, Liège, B-4000 Belgique.

4. Servicio de Investigación Agroalimentaria, Zaragoza, Espagne.

5. Faculté des Sciences, Annaba, Algérie.

$\S$ Ce travail est dédié au Prof. Guellati, directeur de cette recherche, malheureusement décédé le 26 mai 2006.

* Auteur pour la correspondance

Laboratoire d'endocrinologie et de reproduction animale, faculté de Médecine vétérinaire, université de Liège, B-4000, Belgique.

Tél. : +32 (0)43664161; fax : +32 (0)43664165

E-mail : jfbeckers@ulg.ac.be
L'existence de protéines spécifiques (Pspb) ou associées à la gestation (PAG) détectables dans la circulation périphérique maternelle dès le premier mois de la gravidité a été décrite pour la première fois chez la brebis dans les années 1990 (19, 33, 36). Les protéines associées à la gravidité sont synthétisées par les cellules binucléées du trophoblaste des ruminants dès l'implantation jusqu'à la mise bas. Chez l'espèce ovine, ces protéines peuvent être détectées dans la circulation périphérique maternelle dès les dix-huitième à vingtième jours après la fécondation jusqu'au premier mois après l'agnelage (19). Selon plusieurs auteurs, l'évolution de la concentration plasmatique de la PAG peut être influencée par plusieurs facteurs, comme la race $(8,19)$, la masse placentaire totale $(33)$, le nombre de foetus (20) et le stade de la gravidité (19). Etant donné sa spécificité et sa relation directe avec la gravidité, le dosage de la PAG par méthode radio-immunologique (RIA) s'est avéré bénéfique à plusieurs titres, comme la réalisation des réformes appropriées dans les troupeaux suite à un diagnostic précoce de non gravidité (15) et comme paramètre indicatif d'éventuelles pertes de gestation, sous forme de mortalités embryonnaires ou d'avortements $(26,34,35)$.

L'objectif de la présente étude a été la caractérisation des profils plasmatiques de la PAG et de la progestérone au cours de la gravidité et deux semaines après le part chez la brebis Ouled Djellal 
ayant des gestations simples et multiples. En outre, la description individuelle des concentrations plasmatiques chez des brebis ayant subi différents événements pathologiques au cours de la gravidité (perte embryonnaire, avortement et momification) a été retracée.

\section{MATERIEL ET METHODES}

\section{Zone expérimentale}

La présente étude a été menée dans la région de Guelma, est de l'Algérie ( $36^{\circ} 28^{\prime}$ de lat. $\mathrm{N}$. et $7^{\circ} 28^{\prime}$ ' de long. E.). Les animaux ont été élevés dans un système semi-intensif (bergerie), dans une même exploitation. L'alimentation a été basée sur pâturage en vert à volonté et paille de blé. Cette ration a été complétée par de l'orge en grains concassés 45 jours avant et après la lutte, et durant 60 jours avant la date prévue d'agnelage. L'eau a été disponible à volonté. Les femelles ont été vaccinées contre la clavelée (vaccin anticlaveleux, 1,0 ml/tête) et vermifugées (ivermectine, $1 \mathrm{ml} / 50 \mathrm{~kg}$ ) 45 jours avant la lutte.

\section{Les animaux}

Un effectif de 25 brebis multipares de race Ouled Djellal, d'âge compris entre 2,5 et 4 ans, d'un poids vif moyen de $47,4 \pm 4,9 \mathrm{~kg}$ et de note d'état corporel moyenne de $3,1 \pm 0,5$ a été utilisé. Toutes les femelles ont eu un intervalle entre l'agnelage et la lutte supérieur ou égal à 90 jours. Les brebis ont été isolées des mâles pendant au moins 60 jours avant la lutte.

\section{Synchronisation des chaleurs}

L'ensemble des brebis ont été synchronisées entre mars et juillet 2004 au moyen de traitements hormonaux associés à l'effet bélier. Des éponges vaginales imprégnées de $40 \mathrm{mg}$ d'acétate de fluorogestone (FGA, Chronogest ${ }^{\circledR}$, Intervet, France) ont été placées pendant 13 jours. Une injection intramusculaire de 500 UI de Pmsg (pregnant mare serum gonadotropin; Folligon ${ }^{\circledR}$, Intervet) a été administrée à chaque femelle au moment du retrait des éponges.

\section{La lutte}

La lutte de mode naturel et contrôlé a été réalisée par trois béliers adultes de la même race à deux périodes : $48 \mathrm{~h}$ (premier accouplement) et entre 56 et $60 \mathrm{~h}$ (deuxième accouplement) après le retrait des éponges. Les mâles ont été réintroduits 15 jours après la lutte principale, pour détecter et lutter les femelles ayant manifesté un retour en chaleur.

\section{Prise de sang et collecte des échantillons de plasma}

Des prises de sang hebdomadaires ont été réalisées entre la première semaine après la lutte jusqu'à la deuxième semaine post-partum, excepté pour la prise de la deuxième semaine après la lutte, réalisée à 17 jours.

Les prélèvements de sang ont été faits au moyen de seringues jetables dans des tubes à Edta de $5 \mathrm{ml}$. Les tubes ont été immédiatement centrifugés (1 $500 \mathrm{x} g$ pendant $12 \mathrm{~min}$ ) et les échantillons de plasma ont été transvasés et transportés dans une glacière jusqu'à leur lieu de stockage pour être conservés à $-20{ }^{\circ} \mathrm{C}$ jusqu'à leur dosage.

\section{Dosages radio-immunologiques}

Les dosages radio-immunologiques (RIA) de la progestérone et de la PAG ont été réalisés au laboratoire d'endocrinologie et de reproduction animale (faculté de Médecine vétérinaire, université de Liège, Belgique). Les prises de sang des femelles vides ou non fécondées, ainsi que de celles ayant perdu leurs embryons ou ayant avorté ont été analysées à nouveau pour la détermination des concentrations plasmatiques de l'hormone lutéinisante (LH).

\section{Progestérone}

Les concentrations plasmatiques en progestérone ont été déterminées par une méthode RIA, dite directe, sans extraction de l'hormone, basée sur l'utilisation d'un agent bloquant la liaison stéroïde protéine de transport, tel l'ANS (acide sulfonique 8-anilino-1naphtalène (Fluka and Riedel-de Haën, Schweiz, Suisse) (22).

La courbe standard de la progestérone (Sigma Chemical, St Louis, MO, USA) a été constituée de huit points (20, 10, 5, 2,5, 1,2, 0,6, 0,3 et $0,15 \mathrm{ng} / \mathrm{ml}$ ), dilués deux à deux dans du sérum sans stéroïdes, à partir du point le plus haut de la courbe $(20 \mathrm{ng} / \mathrm{ml})$. Le traceur (P4-11-hemisuccinate-2[ $\left.{ }^{125} \mathrm{I}\right]$-iodohistamine ; Amersham Biosciences, Uppsala, Suède) a été dilué dans le tampon salin phosphate $(0,10 \mathrm{M}, \mathrm{pH} 7,0)$ de façon à obtenir une radioactivité d'environ $20000 \mathrm{cpm} / 100 \mu \mathrm{l}$. L'anticorps antiprogestérone (AS\#43) a été utilisé à une dilution initiale de 1:15 000. Le système précipitant contenant le deuxième anticorps a été composé d'anticorps de mouton antigammaglobuline de lapin à 0,87 p. 100 volume : volume, de sérum normal de lapin à 0,17 p. 100 volume : volume, de polyéthylène glycol 6000 à $20 \mathrm{mg} / \mathrm{ml}$ (Vel, Leuven, Belgique), de cellulose microcristalline à $0,05 \mathrm{mg} / \mathrm{ml}$ (Merck, Darmstadt, Allemagne) et de BSA (albumine de sérum bovin) à $2 \mathrm{mg} / \mathrm{ml}$ (Biochemicals, Aurora, OH, USA) solubilisés dans du tampon phosphate. La sensibilité du dosage RIA de la progestérone a été de $0,2 \mathrm{ng} / \mathrm{ml}$.

La technique utilisée a été récemment décrite par Faye et coll. (7) et peut être résumée comme suit : $50 \mu \mathrm{l}$ de chaque échantillon plasmatique ainsi que des différents points de dilution de la courbe standard ont été prélevés en duplicate dans des tubes en polystyrène à fond conique. Ensuite, $300 \mu \mathrm{l}$ de tampon citrate-ANS $(0,10 \mathrm{M}$ citrate, 0,25 p. $100 \mathrm{BSA}$ contenant $100 \mu \mathrm{l}$ d'ANS, pH 4,0), $100 \mu \mathrm{l}$ de traceur et $100 \mu \mathrm{l}$ de premier anticorps antiprogestérone dilué à 1:15 000 ont été successivement ajoutés dans chaque tube. Le tout a été agité et incubé pendant $4 \mathrm{~h}$ à $4{ }^{\circ} \mathrm{C}$. Chacun des points standard et d'échantillons a été ensuite additionné de 1,0 $\mathrm{ml}$ de la solution contenant le deuxième anticorps, avant d'être laissé en incubation pendant $30 \mathrm{~min}$ à température ambiante. Enfin, 2,0 ml de tampon phosphate (tampon RIA stéroïde) ont été ajoutés à tous les tubes, lesquels ont été ensuite centrifugés à $1500 \mathrm{x} g$ pendant 20 min et à $10^{\circ} \mathrm{C}$. A l'issue de cette opération, le surnageant a été éliminé et les tubes ont été décantés avant la lecture de la radioactivité du précipité dans un compteur Multigamma (LKB Wallac 126 Multigamma counter, Turku, Finlande).

\section{Protéine associée à la gestation}

Les concentrations plasmatiques en PAG ont été déterminées par un RIA semi-homologue, avec de la PAG bovine $67 \mathrm{kDa}$ $($ boPAG $67 \mathrm{kDa}$ ) (37) comme standard et traceur, et un anticorps anti-PAG ovines $55 \mathrm{kDa}$ et $59 \mathrm{kDa}$ (ovPAG $57+59 \mathrm{kDa}$; AS\#780) (6) comme anticorps primaire (dilution initiale 1:40 000). Les dosages de PAG ont été réalisés dans du tampon Tris-BSA $(0,025 \mathrm{M}$ de Tris, $0,01 \mathrm{M}$ de $\mathrm{MgCl}_{2}, 0,02$ p. 100 poids : volume de $\mathrm{NaN}_{3}$, pH $7,5)$ contenant $1,0 \mathrm{mg} / \mathrm{ml}$ de BSA. Les points de la courbe standard ont varié de 0,4 à $100 \mathrm{ng} / \mathrm{ml}$. Le traceur $\left({ }^{125} \mathrm{I}-\mathrm{PAG}\right)$ a été marqué par la méthode à la chloramine $\mathrm{T}$ (10) et a subi une dilution appropriée de manière à obtenir une radioactivité de $25000 \mathrm{cpm} / 100 \mu \mathrm{l}$. Le système précipitant contenant le deuxième anticorps a été similaire à celui utilisé pour la progestérone, les différents composants ayant été dilués dans du tampon Tris. La sensibilité du dosage RIA de la PAG a été de $0,4 \mathrm{ng} / \mathrm{ml}$. 
Succinctement, les échantillons de plasma de brebis $(100 \mu \mathrm{l})$ ainsi que les différentes dilutions de la courbe standard $(100 \mu l)$ ont été additionnés à des tubes coniques contenant respectivement 200 et $100 \mu \mathrm{l}$ de Tris-BSA. Ensuite, les tubes contenant les différents points de dilution de la courbe standard ont été additionnés de $100 \mu \mathrm{l}$ de sérum de brebis sans PAG. Finalement, $100 \mu \mathrm{l}$ de traceur et $100 \mu \mathrm{l}$ d'antisérum dilué (1:40 000) ont été ajoutés à tous les tubes, à l'exception de ceux mesurant la liaison non spécifique (NSB pour non-specific binding), lesquels n'ont pas reçu de l'antisérum. Les tubes ont été vortexés et ensuite incubés toute une nuit à température ambiante. Le lendemain, $1,0 \mathrm{ml}$ du système précipitant contenant le deuxième anticorps a été ajouté. L'ensemble a été incubé pendant $30 \mathrm{~min}$ avant l'addition de 2,0 $\mathrm{ml}$ de tampon TrisBSA. Une centrifugation à $1500 \mathrm{x} g$ pendant $20 \mathrm{~min}$ a été effectuée afin de permettre une bonne séparation des fractions libres et liées. Le surnageant a été écarté et la radioactivité du culot mesurée à l'aide d'un compteur Multigamma.

\section{Hormone lutéinisante}

La concentration plasmatique de la LH a été déterminée par RIA, selon la méthode récemment décrite par Zongo et coll. (article soumis pour publication). La LH d'origine porcine (pLH) a été marquée par la méthode à la chloramine $\mathrm{T}$ (10) et a subi une dilution appropriée de manière à obtenir une radioactivité de $25000 \mathrm{cpm} / 100 \mu \mathrm{l}$. Un anticorps anti-LH ovine (anti-oLH; AS\#L34) a été utilisé à une dilution initiale de 1:100 000. La sensibilité du dosage RIA de la $\mathrm{LH}$ a été de $0,5 \mathrm{ng} / \mathrm{ml}$.

\section{Analyse des données}

La taille de la portée, le sexe et le poids vif des agneaux ont été enregistrés à l'agnelage. Une durée moyenne de gravidité de 21 semaines a été utilisée pour l'élaboration des profils en PAG et en progestérone. Tous les résultats ont été exprimés en « $\mathrm{ng} / \mathrm{ml}$ » de plasma et les moyennes des données ont été calculées avec leur erreur standard (ES). Toutes les analyses ont été effectuées avec le modèle mixte de la procédure du modèle linaire généralisé (GLM) du logiciel SAS (23). La semaine de la gravidité et le nombre de fœus ont été considérés comme les principaux facteurs de variation. La différence statistique a été déclarée à $\mathrm{P}<0,05$.

\section{RESULTATS}

Parmi les 25 brebis luttées, 18 sont devenues gravides dès le premier cycle et sont arrivées au terme de la gravidité après une durée moyenne de 150,6 $\pm 2,8$ jours. Deux femelles n'ont pas été fécondées et cinq autres ont eu leur profil décrit individuellement du fait que deux d'entre elles ont eu des mortalités embryonnaires, deux autres ont connu des avortements et la dernière a eu une mortalité embryonnaire suivie d'une fécondation après un accouplement non programmé. De même, les profils de deux brebis ayant donné naissance chacune à un agneau vivant et expulsé un ou deux fœtus momifiés au moment de l'agnelage ont été aussi décrits à part.

Des dix-huit brebis ayant agnelé, douze ont donné naissance à un seul agneau et six ont eu des naissances doubles. Le poids moyen des agneaux issus de gravidités simples et multiples a été respectivement de 4,3 $\pm 0,2$ et $3,4 \pm 0,1 \mathrm{~kg}$.

\section{Profils hormonaux moyens de la PAG et de la progestérone}

Chez deux femelles non fécondées, les concentrations en PAG ont été indétectables et celles en progestérone sont restées au-dessous de $1,0 \mathrm{ng} / \mathrm{ml}$, valeur considérée comme seuil pour établir un diagnostic de gravidité. Ces concentrations en PAG et en progestérone ont été significativement influencées par le stade de la gravidité $(\mathrm{P}<0,0001)$ et non par le nombre de fœtus. Les profils plasmatiques de PAG et de progestérone au cours de la gravidité et des deux premières semaines post-partum chez 18 brebis de race Ouled Djellal sont illustrés dans la figure 1.

Les concentrations en PAG, au départ indétectables, ont augmenté de $0,4 \pm 0,1 \mathrm{ng} / \mathrm{ml}$ au dix-septième jour à $5,9 \pm 1,0 \mathrm{ng} / \mathrm{ml}$ à la $3^{\mathrm{e}}$ semaine de gravidité. Ensuite, une augmentation significative a été observée à la $4^{\mathrm{e}}$ semaine $(30,5 \pm 2,9 \mathrm{ng} / \mathrm{ml} ; \mathrm{P}<0,0001)$, suivie d'une seconde augmentation à la $8^{\mathrm{e}}$ semaine $(45,0 \pm 3,7 \mathrm{ng} / \mathrm{ml}$; $\mathrm{P}<0,05)$. Les concentrations en PAG ont diminué graduellement entre la $9^{\mathrm{e}}$ et la $14^{\mathrm{e}}$ semaine, pour reprendre leur évolution ascendante entre la $16^{\mathrm{e}}$ et la $17^{\mathrm{e}}$ semaine $(47,8 \pm 5,3$ à 66,3 $\pm 10,1 \mathrm{ng} / \mathrm{ml} ; \mathrm{P}<0,01)$, et ensuite entre la $18^{\mathrm{e}}$ et la $19^{\mathrm{e}}$ semaine $(71,8 \pm 15,7$ à $93,7 \pm 23,9 \mathrm{ng} / \mathrm{ml} ; \mathrm{P}<0,05)$. Enfin, une légère diminution des concentrations a été observée à la $20^{\mathrm{e}}$ semaine $(83,8 \pm 15,5 \mathrm{ng} / \mathrm{ml})$, suivie d'une augmentation significative, pour atteindre le pic de sécrétion à la $21^{\mathrm{e}}$ semaine $(116,0 \pm 20,6 \mathrm{ng} / \mathrm{ml}$; $\mathrm{P}<0,005)$. Les concentrations en PAG ont chuté rapidement après l'agnelage, atteignant $36,1 \pm 5,7 \mathrm{ng} / \mathrm{ml}$ à la $1^{\text {re }}$ semaine postpartum et $13,8 \pm 2,5 \mathrm{ng} / \mathrm{ml}$ à la $2^{\mathrm{e}}$ semaine.

Le changement en concentrations de progestérone a été moins marqué (figure 1). Les concentrations ont augmenté graduellement pendant la gravidité, atteignant le niveau maximal à la $17^{\mathrm{e}}$ semaine après saillie $(10,9 \pm 1,1 \mathrm{ng} / \mathrm{ml})$. Ensuite, les concentrations ont décru graduellement, avec un déclin très significatif $(\mathrm{P}<0,0001)$ entre la $20^{\mathrm{e}}(8,5 \pm 0,8 \mathrm{ng} / \mathrm{ml})$ et la $21^{\mathrm{e}}$ semaines $(5,2 \pm 0,9 \mathrm{ng} / \mathrm{ml})$. Après l'agnelage, les concentrations en progestérone ont atteint leurs niveaux basaux dès la $1^{\text {re }}$ semaine après le part $(0,1 \pm 0,2 \mathrm{ng} / \mathrm{ml})$.
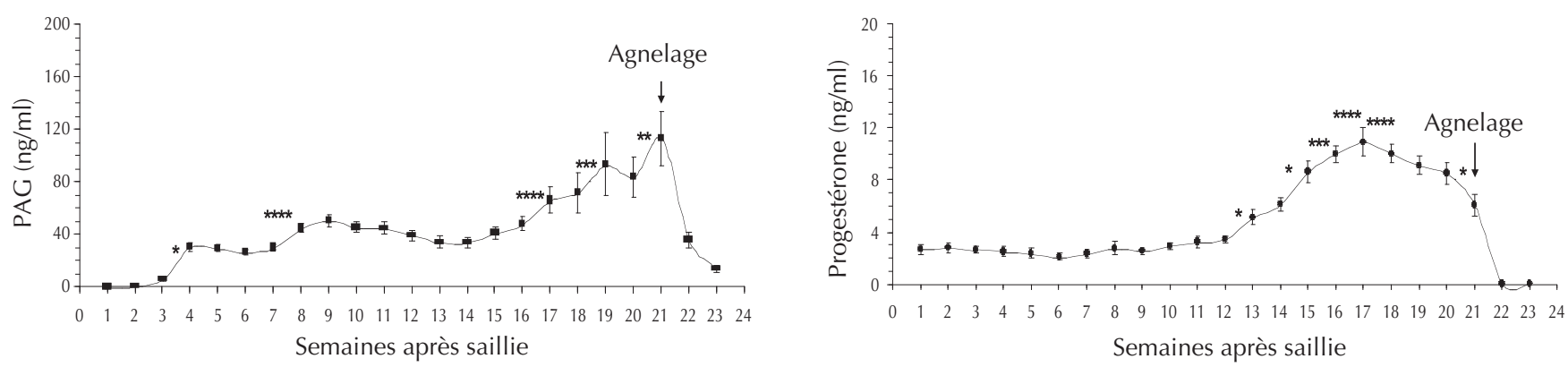

Figure 1 : profils plasmatiques (moyenne $\pm E S$ ) de la progestérone et de la protéine associée à la gestation (PAG) chez la brebis Ouled Djellal $(n=18)$ durant la gravidité et la période post-partum. ${ }^{*} P<0,0001 ;{ }^{* *} P<0,005 ;{ }^{* * *} P<0,01 ;{ }^{* * * *} P<0,05$. 


\section{Profils hormonaux de la PAG et de la progestérone selon la taille de la portée}

Les concentrations en PAG chez les brebis ayant eu des gestations multiples ont été légèrement supérieures à celles observées chez les femelles ayant eu des gestations simples (figure 2). Un écart positif des concentrations en faveur des portées doubles a été observé à la $4^{\mathrm{e}}$ semaine $(25,3 \pm 2,1$ vs $41,3 \pm 5,6 \mathrm{ng} / \mathrm{ml})$, ainsi qu'entre les $16^{\mathrm{e}}$ $(43,7 \pm 6,8$ vs $55,9 \pm 8,2 \mathrm{ng} / \mathrm{ml})$ et $19^{\mathrm{e}}$ semaines $(82,6 \pm 19,8$ vs $115,8 \pm 63,0 \mathrm{ng} / \mathrm{ml})$. Cependant, la comparaison des concentrations semaine par semaine n'a révélé aucune différence significative tout au long de la gravidité.

Une tendance similaire des concentrations plus élevées en progestérone chez les brebis ayant eu des gestations multiples a été observée entre la $13^{\mathrm{e}}$ et la $19^{\mathrm{e}}$ semaine après saillie. En effet, chez les brebis ayant eu des gestations simples, les concentrations en progestérone ont augmenté de 4,5 $\pm 0,4$ ( $13^{\mathrm{e}}$ semaine) à $8,5 \pm 0,9$ ( $17^{\mathrm{e}}$ semaine), tandis que pendant la même période les concentrations ont augmenté de $6,6 \pm 1,3$ à 10,3 $\pm 1,2 \mathrm{ng} / \mathrm{ml}$ chez les femelles ayant eu des gestations multiples $(\mathrm{P}<0,0005$ et $\mathrm{P}<0,005$ respectivement à la $13^{\mathrm{e}}$ et $17^{\mathrm{e}}$ semaine).

\section{Profils des PAG, progestérone et $L H$ lors des différents événements physiopathologiques identifiés au cours de la gravidité}

Les observations cliniques associées à une analyse détaillée des profils en PAG, progestérone et LH ont révélé que deux brebis n'avaient pas été fécondées ( ${ }^{\circ} 1005$ et 1029 ). Sept autres femelles ont été identifiées comme des cas particuliers ; leurs profils hormonaux ont été regroupés en quatre catégories distinctes : brebis ayant perdu leurs embryons ( $\mathrm{n}^{\circ} 1007$ et 1013 ), brebis ayant avorté $\left(n^{\circ} 2025\right.$ au $66^{\mathrm{e}}$ jour et $n^{\circ} 3023$ au $110^{\mathrm{e}}$ jour), brebis ayant eu une perte embryonnaire suivie d'une fécondation après un accouplement non programmé $\left(n^{\circ} 1025\right)$ et brebis ayant donné naissance à un agneau vivant avec un $\left(\mathrm{n}^{\circ} 1037\right)$ et deux fœus momifiés $\left(n^{\circ} 1007\right)$.

\section{Brebis ayant perdu leurs embryons}

Chez cette catégorie de brebis (figure 3), les concentrations en progestérone ont été supérieures au seuil utilisé pour le diagnostic de gravidité au dix-septième jour de gravidité ( 1,2 et $4,4 \mathrm{ng} / \mathrm{ml}$, respectivement chez les brebis n ${ }^{\circ} 1007$ et 1013 ). Cependant, chez la brebis $n^{\circ} 1007$, les concentrations en cette hormone sont restées inférieures à $1,2 \mathrm{ng} / \mathrm{ml}$ entre la $3^{\mathrm{e}}$ et la $5^{\mathrm{e}}$ semaine après la lutte. Chez

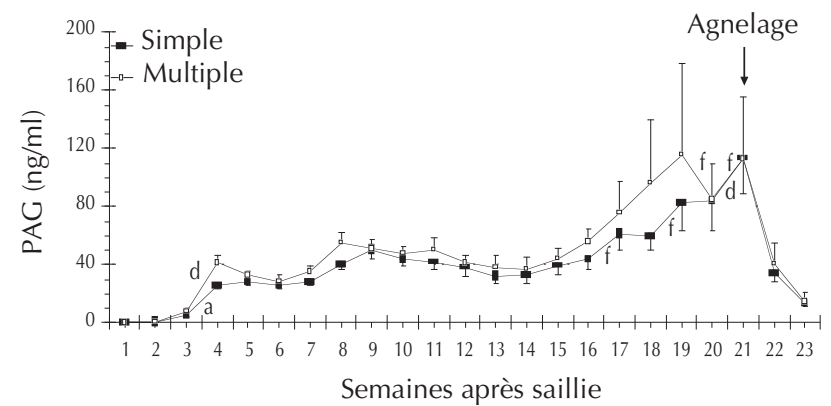
ques ( $\left.{ }^{*} P<0,0005 ;{ }^{* *} P<0,005\right)$. des concentrations en PAG respectivement à la $4^{\mathrm{e}}$ semaine (respectivement 12,6 et 20,0 ng/ml) et par les niveaux basaux en LH. Des chutes brutales des concentrations en progestérone, et plus particulièrement en PAG, ont été remarquées entre les $5^{\mathrm{e}}$ et $6^{\mathrm{e}}$ semaines de gravidité pour les deux brebis, indiquant ainsi les pertes embryonnaires.

La reprise de la cyclicité a été caractérisée par l'augmentation de la progestérone dès la $14^{\mathrm{e}}$ semaine après saillie pour la brebis $\mathrm{n}^{\circ} 1007$ et dès la $9^{\mathrm{e}}$ semaine pour la brebis $\mathrm{n}^{\circ} 1013$. L'alternance des pics de LH et la persistance du niveau basal de la protéine placentaire (PAG) vérifiée pendant la période d'observation ont confirmé cette situation.

\section{Brebis ayant avorté}

Ce groupe concerne deux brebis, ayant avorté à $66\left(n^{\circ} 2025\right)$ et 110 jours $\left(n^{\circ} 3023\right)$ après saillie (figure 3 ). Il est à remarquer que chez la brebis $n^{\circ} 2025$ la concentration plasmatique en progestérone a été de 1,0 ng/ml au vingt et unième jour. Ensuite, cette concentration a chuté, restant inférieure à $0,9 \mathrm{ng} / \mathrm{ml}$ entre la $4^{\mathrm{e}}$ et la $9^{\mathrm{e}}$ semaine de gravidité, moment probable de la mort fotale. Chez la brebis $n^{\circ} 3023$, les concentrations en progestérone ont été considérées comme normales jusqu'à la $15^{\mathrm{e}}$ semaine après saillie. Les avortements ont été confirmés par l'expulsion des fœtus ainsi que par la chute des concentrations de la PAG à la $9-10^{\mathrm{e}}$ semaine pour la première $\left(\mathrm{n}^{\circ} 2025\right)$ et à la $15-16^{\mathrm{e}}$ semaine pour la seconde $\left(\mathrm{n}^{\circ}\right.$ $3023)$.

\section{Brebis ayant eu une perte embryonnaire précoce suivie d'une fécondation après un accouplement non programmé}

L'analyse des profils plasmatiques chez la brebis $\mathrm{n}^{\circ} 1025$ a révélé un cas particulier dans lequel une gestation est décelée avec certitude, annoncée par l'augmentation des concentrations en PAG entre la $3^{\mathrm{e}}(4,7 \mathrm{ng} / \mathrm{ml})$ et la $6^{\mathrm{e}}$ semaine après la première saillie $(23,7 \mathrm{ng} / \mathrm{ml})$. Des concentrations en progestérone entre 1,1 et $2,6 \mathrm{ng} / \mathrm{ml}$, et les faibles concentrations en LH pendant cette même période ont confirmé le déroulement de la gravidité. Une chute des concentrations en PAG $(0,5 \mathrm{ng} / \mathrm{ml})$ et en progestérone $(0,5 \mathrm{ng} / \mathrm{ml})$ est intervenue ensuite à la $7^{\mathrm{e}}$ semaine de gravidité, indiquant une perte embryonnaire. Ceci a été suivi par une deuxième période d'augmentation des concentrations en PAG et en progestérone (entre la $9^{\mathrm{e}}$ et la $10^{\mathrm{e}}$ semaine), indiquant le début d'une nouvelle gravidité. En effet, comme illustré dans la figure 4, des concentrations élevées en PAG et en progestérone ont été trouvées décalées de 7-8 semaines par rapport à la première saillie. Effectivement, cette brebis a donné naissance à deux agnelles vivantes quelques semaines après la fin du calendrier des prélèvements.

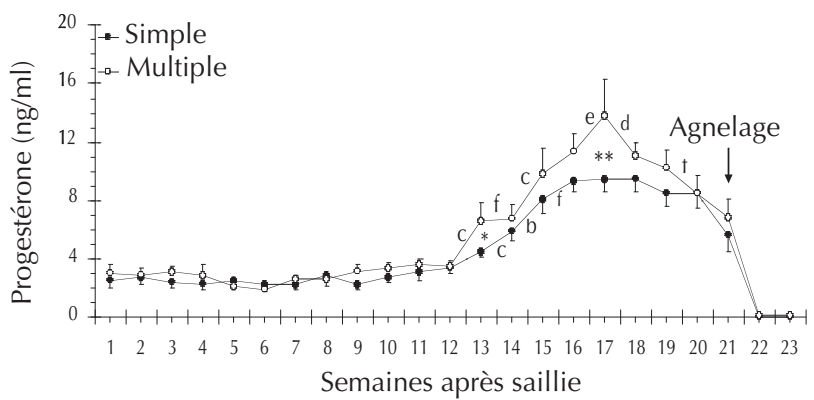

Figure 2 : profils plasmatiques (moyenne $\pm E S$ ) de la progestérone et de la protéine associée à la gestation (PAG) chez la brebis Ouled Djellal ayant des gestations simples $(n=12)$ et multiples $(n=6)$. Les différences de concentrations entre différentes semaines dans un même groupe sont indiquées par des lettres $\left({ }^{a} P<0,0001 ;{ }^{b} P<0,0005 ;{ }^{c} P<0,001 ; d P<0,005\right.$; e $\left.P<0,001 ;{ }^{f} P<0,05\right)$. Les différences de concentrations entre gestations simples et multiples sont indiquées par des astéris- 


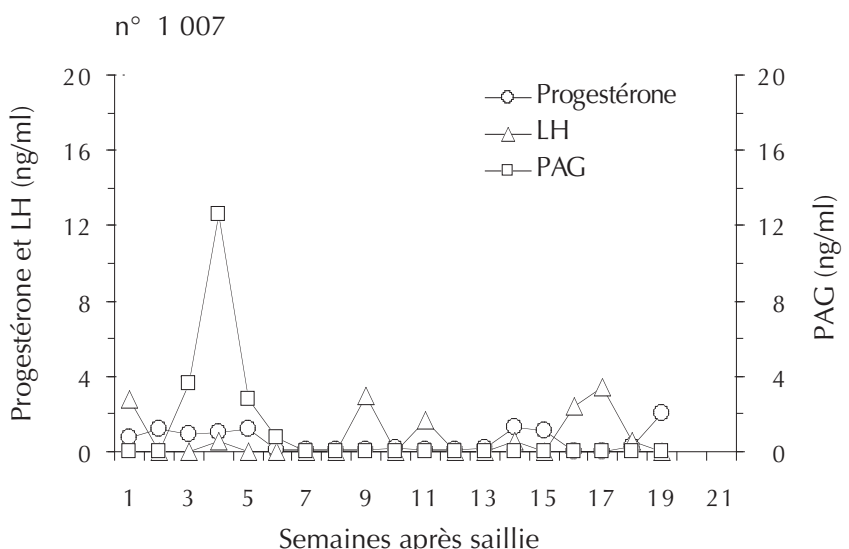

$n^{\circ} 2025$

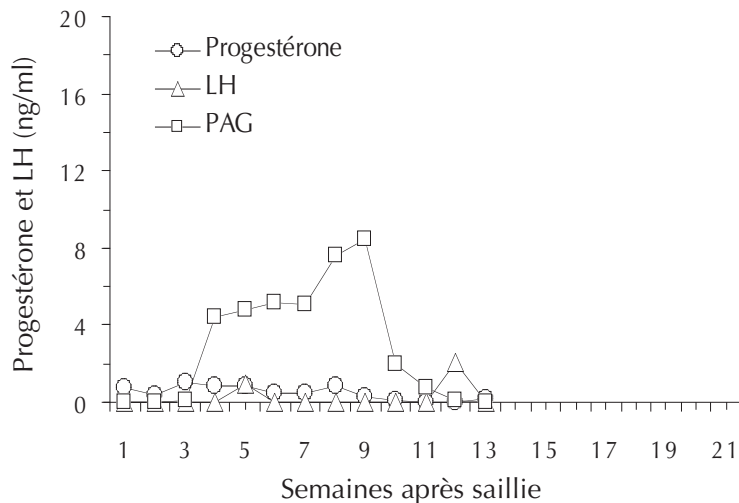

$n^{\circ} 1013$

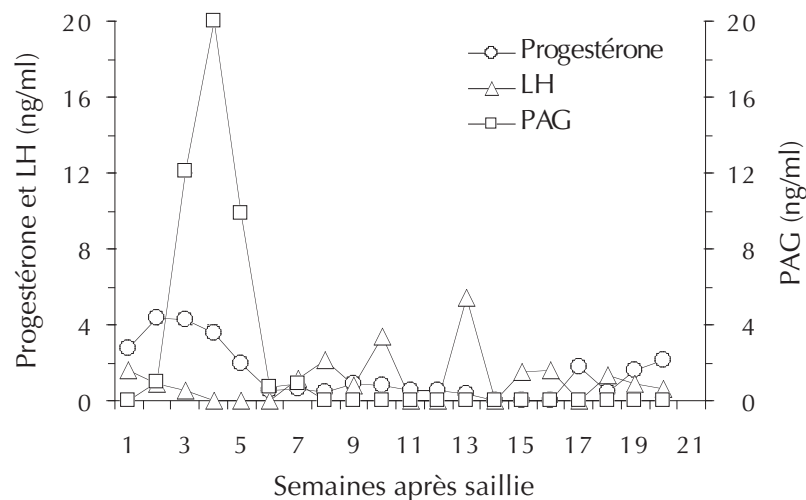

$n^{\circ} 3023$

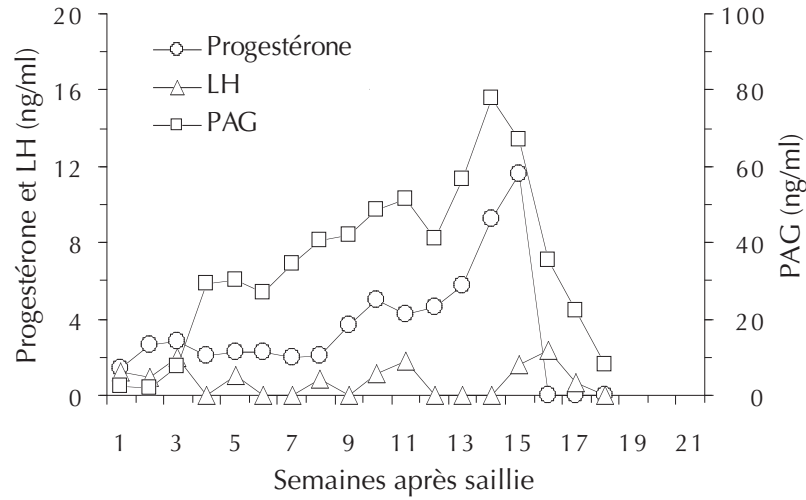

Figure 3 : profils plasmatiques de la progestérone, de l'hormone lutéinisante (LH) et de la protéine associée à la gestation (PAG) chez des brebis Ouled Djellal ayant perdu leurs embryons au premier mois de gravidité ( $n^{\circ} 1007$ et 1013 ) ou ayant avorté aux $66^{\mathrm{e}}\left(n^{\circ} 2025\right)$ et $110^{\mathrm{e}}$ jours après saillie $\left(n^{\circ} 3023\right)$.

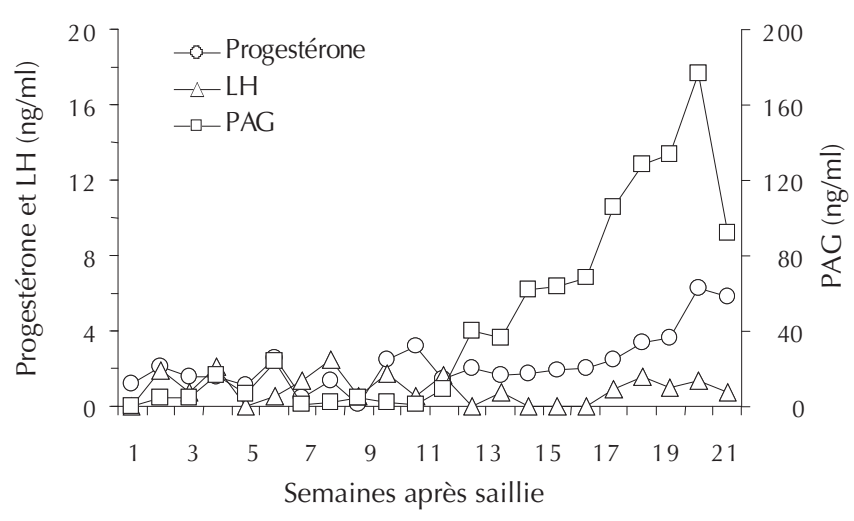

Figure 4 : profils plasmatiques de la progestérone, de l'hormone lutéinisante $(L H)$ et de la protéine associée à la gestation (PAG) chez une brebis Ouled Djellal ayant perdu son(ses) embryon(s) et ayant été fécondée par la suite accidentellement ( $n^{\circ} 1$ 025), probablement à la $9^{e}$ semaine.

\section{Brebis ayant donné naissance à un agneau vivant et expulsé un ou deux foetus momifiés}

Ce groupe, qualifié aussi de particulier, a concerné deux brebis, ayant mis bas à terme un agneau vivant et normal avec un $\left(n^{\circ} 1037\right)$ et deux $\left(n^{\circ} 1003\right)$ fœtus momifiés (figure 5). Selon leur profil, il ressort que la concentration plasmatique en PAG a été plus importante aussi bien à la $3^{\mathrm{e}}$ qu'entre la $7^{\mathrm{e}}$ et la $14^{\mathrm{e}}$ semaine de gravidité chez la brebis $n^{\circ} 1003$. Au début, cette dernière était porteuse de trois embryons/fœetus, tandis que la brebis $n^{\circ} 1037$ était initialement porteuse de deux embryons/fœtus. A l'opposé, les concentrations en progestérone ont été plus élevées chez la brebis ayant eu un seul agneau momifié que chez celle en portant deux.

La mort des foetus momifiés a probablement eu lieu entre les $3^{\mathrm{e}}$ et $4^{\mathrm{e}}$ mois de gravidité aussi bien chez la brebis $\mathrm{n}^{\circ} 1003$ (portant deux fœetus momifiés) que chez la brebis $n^{\circ} 1037$ (portant un fœetus momifié), comme cela a pu être estimé à partir de la taille des fœtus momifiés observés après l'agnelage. Cette situation a également été remarquée par les changements même moins importants révélés dans les concentrations plasmatiques, particulièrement en PAG entre les $15^{\mathrm{e}}$ et $18^{\mathrm{e}}$ semaines de gravidité (figure 5).

\section{DISCUSSION}

Chez les femelles gravides, les concentrations plasmatiques en PAG et en progestérone sont restées élevées à partir de la $3^{\mathrm{e}}$ semaine après la fécondation, dépassant les seuils discriminatoires de $1 \mathrm{ng} / \mathrm{ml}$ pour la progestérone (27) et de 1 à $2 \mathrm{ng} / \mathrm{ml}$ pour la PAG $(15,31)$. Chez deux femelles seulement, les concentrations en PAG (et en progestérone) ont été en dessous des seuils établis pour le diagnostic de gravidité. En effet, la sécrétion de la PAG par les cellules binucléées du trophoblaste, bien que faible au départ, devient plus importante à partir des $22^{\mathrm{e}}-24^{\mathrm{e}}$ jours de gravidité (15). 
$\mathrm{n}^{\circ} 1037$

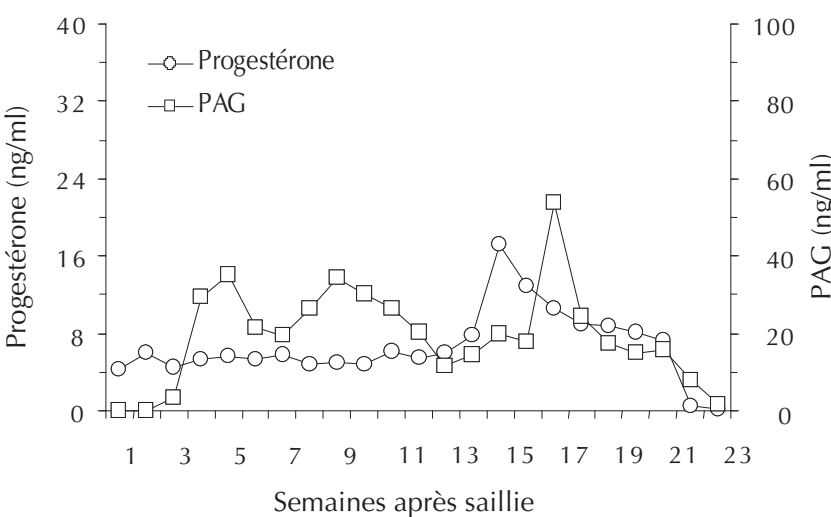

$\mathrm{n}^{\circ} 1003$

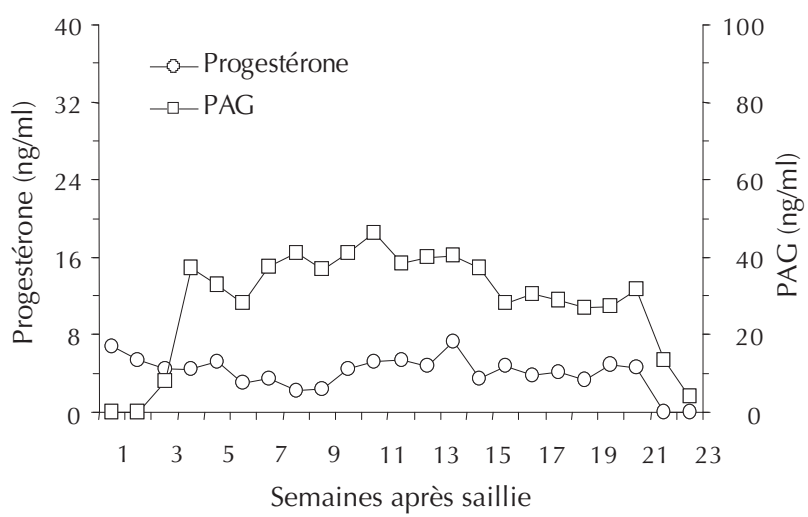

Figure 5 : profils plasmatiques de la progestérone et de la protéine associée à la gestation (PAG) chez deux brebis Ouled Djellal ayant chacune mis bas à un agneau vivant et à un ( $\left.n^{\circ} 1037\right)$ et deux ( $\left.n^{\circ} 1003\right)$ fotus momifiés.

En parallèle, la sécrétion de progestérone est nécessaire pour assurer un environnement utérin favorable à la survie de l'embryon (27).

\section{Profil hormonal moyen de la PAG}

Des concentrations plasmatiques en PAG ont été détectables dès le vingt et unième jour de gravidité chez 87,0 p. 100 des brebis Ouled Djellal gravides. A partir du vingt-huitième jour, de telles concentrations ont été détectées chez 100 p. 100 des brebis gravides. Ceci est en adéquation avec les résultats avancés par plusieurs auteurs chez la même espèce et pour différentes races, employant le dosage de la Pspb (32) ou le dosage de la PAG, avec des tests RIA dits hétérologues $(15,19)$ ou homologues $(29,31)$.

Des profils plasmatiques en PAG au cours de la gravidité ont été décrits chez différentes races, comme la Churra et la Mérinos (19), l'Assaf (20), la Berrichonne (8), ainsi que la German Blackheaded Mutton (GBM), la Rhoen et des croisées GBM x Dorper (16). Les concentrations en PAG observées dans la présente étude ont été beaucoup plus basses que celles décrites par Ranilla et coll. (20), et Gajewski et coll. (8), mais fort similaires à celles décrites pour les races allemandes (16). Ces différences en concentration sont vraisemblablement dues à l'utilisation récente d'antisérums produits contre de nouvelles préparations en PAG. En effet, suite à la purification et à la caractérisation de nouvelles PAG chez la chèvre (9) et chez la brebis (6), des antisérums ont été produits chez des lapins. Ces derniers sont actuellement utilisés de manière routinière pour établir le diagnostic de gravidité $(15,29,31)$ ou pour déceler les profils plasmatiques de cette protéine dans des cas de gravidités arrivant à terme (16), ou étant interrompues par des mortalités embryonnaires ou fæatales $(34,35)$.

Chez la race Ouled Djellal, l'évolution des profils en PAG est très similaire à celle décrite chez la brebis Churra et Assaf $(19,20)$, ainsi que chez les races germaniques (16). L'augmentation importante des concentrations plasmatiques en PAG entre la $3^{\mathrm{e}}$ et la $4^{\mathrm{e}}$ semaine de gravidité chez les brebis Ouled Djellal a été un peu plus précoce que celle observée chez les races Mérinos (19) et Berrichonne (8), chez lesquelles l'augmentation initiale des concentrations plasmatiques a été observée à la $5^{\mathrm{e}}$ semaine de gravidité. De même, une augmentation des concentrations à la $9^{e}$ semaine a été observée chez les brebis Ouled Djellal, ainsi que chez les Churra et Mérinos (19). Cette augmentation a été plus précoce que celle décrite à la $11^{\mathrm{e}}$ semaine pour des brebis Berrichonnes (8). Notons au passage qu'un léger déclin des concentrations plasmatiques en PAG a été observé entre la $9^{\mathrm{e}}$ et la $14^{\mathrm{e}}$ semaine de gravidité chez la race Ouled Djellal. Cette réduction est caractéristique de la majorité des races ovines étudiées. L'origine de cette décroissance n'est pas élucidée à l'heure actuelle bien que l'on puisse suspecter l'expression temporaire des différentes formes de PAG moins bien reconnues dans les systèmes RIA utilisés.

Enfin, comme pour les races Churra (19), Assaf (20), GBM, Rhoen et croisées (16), les concentrations maximales de PAG ont été atteintes à la $21^{\mathrm{e}}$ semaine de gravidité, suivies de leur chute pendant les deux premières semaines post-partum. L'augmentation importante des concentrations durant ce dernier stade de la gravidité pourrait être attribuée à une forte migration des cellules binucléées du placenta pendant la fin de la gravidité, ou à une sécrétion accrue due à des transformations tissulaires précédant la mise bas.

Aussi bien chez les brebis que chez les chèvres, la disparition rapide de la PAG après le part est avantageuse et ne constitue pas une entrave dans le diagnostic de gravidité précoce. Cette caractéristique les distingue des bovins chez qui cette disparition n'est totale qu'après 80 à 120 jours post-partum (38), ce qui constitue un problème en cas de saillie intervenant dans les trois premiers mois suivant le vêlage.

\section{Profil hormonal moyen de la progestérone}

L'analyse des concentrations plasmatiques en progestérone au cours de la gravidité fait apparaître que cette hormone stéroïdienne est détectée dès le dix-septième jour de gravidité chez 95,7 p. 100 des brebis Ouled Djellal à une concentration supérieure au seuil, soit une manifestation plus précoce que la PAG. En effet, le profil hormonal de la progestérone a été marqué par une première phase (allant de la $1^{\text {re }}$ jusqu'à la $12^{\mathrm{e}}$ semaine après lutte), caractérisée par une stabilité relative dans les concentrations de la progestérone. Ainsi, ce n'est qu'à l'issue de cette phase que des augmentations significatives des concentrations ont été enregistrées avec la manifestation de l'unique pic de concentration observé à la $17^{\mathrm{e}}$ semaine de gravidité. En effet, l'évolution du profil pour la race Ouled Djellal ne va pas dans le même sens que celles enregistrées chez les races Churra, Mérinos et Assaf $(19,20)$, caractérisées par un pic à la $16^{\mathrm{e}}$ semaine, une chute à la $17^{\mathrm{e}}$ semaine, puis la reprise avec une augmentation jusqu'à la 19-20 e semaine où les valeurs maximales sont atteintes. Bien que les dissemblances de profils à la fin de la gravidité puissent être attribuées à des différences entre races, il n'est pas exclu que l'utilisation d'un système de dosage sans extraction ait pu être à l'origine de ce résultat. 
L'augmentation progressive de la progestérone à partir de la $11-12^{\mathrm{e}}$ semaine de gravidité jusqu'à l'atteinte de son pic à la $17^{e}$ semaine est fort probablement due à l'apparition de la production de cette hormone par le placenta, en plus de son origine ovarienne (17, 28). En effet, selon Denamur et Martinet (3), la progestérone ovarienne n'est indispensable que pendant les deux premiers mois de gravidité. Au-delà, le placenta est capable d'assurer sa synthèse de façon à maintenir la gravidité même s'il y a ovariéctomie (18). Ces observations ont été confirmées par Linzell et Heap (17) qui suggèrent que, chez la brebis, la production de la progestérone par le placenta est cinq fois plus importante que celle produite par l'ovaire.

$\mathrm{Au}$ moment de la parturition et pendant les deux semaines postpartum, une chute brutale des concentrations plasmatiques de la progestérone et de la PAG a été observé. Cette chute a été plus précoce pour la progestérone qui a atteint son niveau basal dès la $1^{\text {re }}$ semaine post-partum, comparée à la PAG restée présente avec de faibles concentrations au-delà de la $2^{\mathrm{e}}$ semaine post-partum. Ceci corrobore les résultats obtenus par Ranilla et coll. $(19,20)$ chez les races Churra, Mérinos et Assaf, ainsi que ceux décrits par Willard et coll. (33) en utilisant un RIA pour la Pspb.

\section{Profils hormonaux de la PAG et de la progestérone selon la taille de la portée}

Des études réalisées par Willard et coll. (33), et Vandaele et coll. (29) paraissent indiquer l'existence d'une relation entre le nombre de fœtus et les concentrations en PAG. En effet, jusqu'à la $15^{\mathrm{e}}$ semaine de gravidité, les concentrations plasmatiques en PAG ont été discrètement plus élevées dans le cas des brebis Ouled Djellal ayant des portées multiples. Bien que les différences en concentration n'aient pas été significatives $(P \geq 0,05)$, ces écarts ont été plus marqués entre la $17^{\mathrm{e}}$ et la $19^{\mathrm{e}}$ semaine, pour ensuite disparaître à la $20-21^{\text {e }}$ semaine. Dans le même ordre d'idées, Ranilla et coll. (20) ont montré que les brebis Assaf gravides de jumeaux ont présenté des concentrations en PAG supérieures depuis la $12^{\mathrm{e}}$ semaine de gravidité jusqu'à la mise bas. Cependant, selon les mêmes auteurs, cette différence n'a été statistiquement significative que durant la $21^{\mathrm{e}}$ semaine de gravidité. Ceci peut être expliqué par une grande variabilité individuelle des concentrations, due à un nombre réduit d'animaux employés (10 brebis Assaf dont 5 portées simples et 5 doubles). En effet, dans une étude récente, Ledezma-Torres et coll. (16) ont pu mettre en évidence des différences significatives entre portées simples $(n=45)$ et multiples $(n=18)$ aussi bien à la $8^{e}$ et $9^{\mathrm{e}}$ semaine, qu'entre la $12^{\mathrm{e}}$ et la $21^{\mathrm{e}}$ semaine de gravidité.

Chez les brebis Ouled Djellal, les concentrations en progestérone ont été plus élevées chez les femelles à portée multiple entre la $13^{\mathrm{e}}$ et $19^{\mathrm{e}}$ semaine de gravidité. Ces résultats sont légèrement différents de ceux décrits par Ranilla et coll. (20) qui ont montré des concentrations de progestérone plus élevées $(\mathrm{P}<0,05)$ chez les brebis Assaf présentant des portées doubles, et ceci entre la $12^{\mathrm{e}}$ et la $20^{\mathrm{e}}$ semaine de gravidité. La même tendance a été signalée par Hall et coll. (11), et par Rhind et coll. (21) suggérant que les niveaux de progestérone adéquats pour le maintien de la gravidité varient avec le nombre de fotus. Il en est de même pour Kalkan et coll. (14) qui ont décrit des concentrations plus élevées de progestérone pendant la deuxième moitié de la gravidité chez les brebis ayant des portées doubles et triples que chez celles ayant des portées simples (respectivement 19,2, 29,9 et 9,2 $\mathrm{ng} / \mathrm{ml}$ ).

Tout comme pour la PAG, le dénombrement des fœus par le dosage de la progestérone chez la race Ouled Djellal doit être envisagé avec précaution. Ainsi, bien que significative, l'interprétation des différences de concentration à la $13^{\mathrm{e}}$ et $17^{\mathrm{e}}$ semaine doit tenir compte de la période exacte de la gravidité ainsi que d'une grande variabilité individuelle.

\section{Identification des différents événements physiopathologiques associés à la gravidité}

Chez deux brebis, les concentrations en progestérone (17 jour) et en PAG ( $21^{\mathrm{e}}$ jour) ont confirmé une gravidité suite à la saillie. Chez l'une d'elles ( $n^{\circ} 1$ 013), les concentrations en PAG et en progestérone ont été considérées comme normales au départ, pour ensuite décroître brutalement dès la $5-6^{\mathrm{e}}$ semaine, indiquant ainsi la survenue de la perte embryonnaire. Chez la brebis $n^{\circ} 1007$, les concentrations en progestérone sont restées faibles pendant les cinq premières semaines de la gravidité, pouvant ainsi suggérer une synthèse insuffisante de progestérone comme étant à l'origine de la mortalité embryonnaire. Ces résultats vont dans le même sens que ceux signalés suite aux dosages de la Pspb et de la progestérone chez les brebis (32) et les chèvres (13), ainsi que de la PAG et de la progestérone chez les chèvres $(34,35)$ et chez les vaches $(25)$. Ces travaux confirment que si le dosage de la progestérone $\left(17^{\mathrm{e}}\right.$ et $21^{\mathrm{e}}$ jours de gravidité) ou de la PAG (à partir du $28^{\mathrm{e}}$ jour) permettent de diagnostiquer une gravidité, leur combinaison, en revanche, permet d'identifier les pertes embryonnaires et d'estimer le moment de leur survenue $(4,12,24,25,26)$. De ce fait, ils permettent de différencier plus précocement des échecs de fécondation, suite à l'insémination artificielle ou à la monte naturelle, de ceux dus à des mortalités embryonnaires.

En outre, les dosages associés de la PAG et de la progestérone ont permis d'élucider l'état physiologique particulier de la brebis $\mathrm{n}^{\circ} 1025$, caractérisé par un trop long intervalle entre la date de fécondation probable et celle de la mise bas. En effet, si des gravidités prolongées ont été déjà décrites dans la littérature (30), des durées de gravidité excessivement longues sont plus souvent le résultat d'une fécondation accidentelle non perçue par l'éleveur lui-même. Dans le présent cas, le décalage des concentrations plasmatiques en PAG et en progestérone par rapport à celui de la moyenne de la race, associé à des concentrations détectables de LH suite à une chute des concentrations en PAG et en progestérone confirment l'hypothèse du commencement d'une nouvelle gestation. La dépression des concentrations en LH lors d'une gestation (1) et de la réapparition de battements au moment de la reprise de la cyclicité ont déjà été décrites dans la littérature (2).

Les avortements survenus aux $66^{\mathrm{e}}$ et $110^{\mathrm{e}}$ jours ont été identifiés cliniquement. Comme cela a été rapporté dans les cas de mortalités embryonnaires, l'analyse rétrospective des concentrations en PAG et en progestérone a établi deux différents cas de figure. Chez la brebis ayant avorté au $66^{\mathrm{e}}$ jour $\left(\mathrm{n}^{\circ} 2025\right)$, les concentrations en progestérone ont été très faibles, restant inférieures à $1,0 \mathrm{ng} / \mathrm{ml}$ jusqu'au moment de l'expulsion du fœtus. Dans le deuxième cas d'avortement, la chute de concentrations en PAG a précédé celle de la progestérone. De même, le déclin des concentrations a précédé l'expulsion du fœtus. Des profils similaires suite à des avortements ont été décrits chez la vache $(4,12,24,26)$, la chèvre $(7,12$, $25,34,35)$ et la brebis $(16,32)$. Des études complémentaires sont nécessaires pour établir si l'altération de la fonction trophoblastique a été la cause ou la conséquence de la détresse et de la mort fœtale.

Cette étude a montré que la présence d'un ou deux fœtus momifiés parallèlement à celle d'un fœus viable n'a eu aucun effet sur la durée de la gravidité. En effet, les deux brebis concernées ont mis bas après 151 et 152 jours de gestation (avec respectivement un et deux fœtus momifiés). La même observation a été signalée chez la chèvre par Zarrouk et coll. $(34,35)$. Cependant, chez les chèvres, la variabilité de la durée de la gravidité a été augmentée. Ainsi, le profil plasmatique de ces deux hormones semble indiquer que, malgré la mort d'un ou de plusieurs fœus, accompagnée d'une réduction des concentrations plasmatiques en PAG et en progestérone, 
le maintien de la gravidité est assuré par le placenta du fœtus vivant. Par ailleurs, le déclenchement de la parturition, processus régi par une cascade hormonale particulière, reste assuré par l'axe hypothalamo-hypophyso-surrénalien du fotus viable.

\section{CONCLUSION}

Le dosage radioimmunologique de la PAG chez la brebis Ouled Djellal a permis de déterminer le profil d'évolution de cette glycoprotéine durant la gravidité et deux semaines après le part, au même titre que le dosage de la progestérone par la méthode dite directe, sans extraction de l'hormone. Ces dosages ont mis en évidence des profils légèrement différents de ceux décrits pour d'autres races ovines, ce qui suggère l'existence de différences interraciales, tant pour la PAG que pour la progestérone. Les concentrations de PAG et de progestérone n'ont pas été affectées par la taille de la portée (nombre de fœtus).

Si le dosage de la progestérone ou de la PAG seul permet de diagnostiquer une gestation respectivement au dix-septième et à partir du vingt-huitième jours après la lutte, leur combinaison, en revanche, est plus avantageuse. Elle permet avec certitude le repérage des femelles vides ou non fécondées après leur lutte ou insémination. De plus, la combinaison des dosages et leur répétition permettent d'identifier et de localiser dans le temps (suivant l'âge de gestation) des accidents de parcours qui peuvent survenir durant la gravidité, tels que les pertes embryonnaires, les avortements et les saillies accidentelles.

Si l'on considère séparément chaque signal (progestérone et PAG) pour l'analyse rétrospective des cas de pertes embryonnaires ou fœtales au cours de la gravidité, il apparaît que la PAG subit une chute de concentration plusieurs jours (voire une semaine) avant la chute du taux de progestérone. Selon ce critère, la $\mathrm{PAG}$ se révèle comme étant un signal plus précoce. Finalement, cette étude a permis d'observer que, malgré une réduction des concentrations plasmatiques de la PAG et de la progestérone, la durée de la gravidité n'a pas été altérée par la présence de fœtus momifiés dans l'utérus chez les brebis portant en parallèle un fœtus vivant.

La réalisation du dosage de la $\mathrm{LH}$ a permis de confirmer certains états physiologiques révélés par les autres dosages : soit par la dépression des concentrations lors d'une gravidité évolutive, soit par sa manifestation sous forme de pics alternés lors de la reprise d'une activité sexuelle chez les femelles non fécondées ayant subi une perte embryonnaire.

\section{Remerciements}

Les dosages RIA ont été réalisés grâce au soutien des fonds spéciaux pour la recherche de l'université de Liège (crédits classiques, 2002). Les auteurs remercient Dr J.L. Hornick pour les conseils et suggestions apportés à l'analyse statistique, et Mme R. FaresNoucairi pour la mise en forme du manuscrit.

\section{BIBLIOGRAPHIE}

1. AL-GUBORY K.H., 1999. Absence of regular pulsatile LH secretion during pre- and post-implantation periods in sheep. Eur. J. Endocrinol., 141: 521-525.

2. AL-GUBORY K.H., HERVIEU J., FOWLER P.A., 2003. Effects of pregnancy on pulsatile secretion of $\mathrm{LH}$ and gonadotrophinreleasing hormone-induced LH release in sheep: a longitudinal study. Reproduction, 125: 347-355.

3. DENAMUR R., MARTINET J., 1955. Effet de l'ovariectomie chez la brebis pendant la gestation. C.R. Soc. Biol., 149 : 2105.

4. DOBSON H., ROWAN T.G., KIPPAX I.S., HUMBLOT P., 1993. Assessment of fetal number, and fetal and placental viability throughout pregnancy in cattle. Theriogenology, 40: 411-425.

5. EL AMIRI B., KAREN A., COGNIE Y., SOUSA N.M., HORNICK J.L., SZENCI O., BECKERS J.F., 2003. Diagnostic et suivi de gestation chez la brebis : réalités et perspectives. Prod. Anim., 16 : 79-90.

6. EL AMIRI B., REMY B., SOUSA N.M., JORIS B., OTTHIERS N.G. PERENYI Z., MBOKO H.B., BECKERS J.F., 2003. Isolation and partial characterization of three pregnancy-associated glycoproteins from the ewe placenta. Mol. Reprod. Dev., 64: 199-206.

7. FAYE D., SULON J., KANE Y., BECKERS J.F., LEAK S., KABORET Y., SOUSA N.M., LOSSON B., GEERTS S., 2004. Effects of an experimental Trypanosoma congolense infection on the reproductive performance of West African Dwarf goats. Theriogenology, 62: 1438-1451.

8. GAJEWSKI Z., BECKERS J.F., SOUSA N.M., THUN R., SULON J., FAUNDEZ R., 1999. Determination of pregnancy-associated glycoprotein concentrations in sheep: a retrospective study. Adv. Cell. Biol., 26: 89-96.

9. GARBAYO J.M., REMY B., ALABART J.L., FOLCH J., WATTIEZ R., FALMAGNE P., BECKERS J.F., 1998. Isolation and partial characterization of a pregnancy-associated glycoprotein family from the goat placenta. Biol. Reprod., 58: 109-115.

10. GREENWOOD F.C., HUNTER W.M., GLOVER J.S., 1963. The preparation of 131-I labeled human growth hormone of high specific radioactivity. Biochemistry, 89: 114-123.

11. HALL D.G., HOST P.J., SHUTT D.A., 1992. The effect of nutritional supplements in late pregnancy on ewe colostrum production plasma progesterone and IGF-1 concentrations. Aust. J. Agric. Res., 43: 337-352.
12. HUMBLOT P., 2001. Use of pregnancy specific proteins and progesterone assays to monitor pregnancy and determine the timing, frequencies and sources of embryonic mortality in ruminants. Theriogenology, 56: 1417-1433.

13. HUMBLOT P., DE MONTIGNY G., JEANGUYOT N., TETEDOIE F., PAYEN B., THIBIER M., SASSER R.G., 1990. Pregnancy-specific protein $B$ and progesterone concentrations in French alpine goats throughout gestation. J. Reprod. Fert., 89: 205-212.

14. KALKAN C., CETIN H., KAYGUSUZOGLU E., YILMAZ B., CIFTCI M., YIDIZ H., YIDIZ A., DEVECI H., APAYDIN A.M., OCAL H., 1996. An investigation on plasma progesterone levels during pregnancy and parturition in the Ivesti sheep. Acta Vet. Hung., 44: 335-340.

15. KAREN A., BECKERS J.F., SULON J., SOUSA N.M., SZABADOS K., RECZIGEL J., SZENCl O., 2003. Early pregnancy diagnosis in sheep by progesterone and pregnancy-associated glycoprotein tests. Theriogenology, 59: 1941-1948.

16. LEDEZMA-TORRES R.A., BECKERS J.F., HOLTZ W., 2006. Assessment of plasma profile of pregnancy-associated glycoprotein (PAG) in sheep with a heterologous (anti-caPAG $(55+59)$ ) RIA and its potential for diagnosing pregnancy. Theriogenology, 66: 906-912.

17. LINZELL J.L., HEAP R.B., 1968. A comparison of progesterone metabolism in the pregnant sheep and goat: source of production and an estimation of uptake by some target organs. J. Endocrinol., 41: 433-438.

18. MEITES J., WEBSTER H.D., YOUNG W., THORP F. JR, HATCH R.N., 1951. Effects of corpora lutea removal and replacement with progesterone on pregnancy in goats. J. Anim. Sci., 10: 411-416.

19. RANILLA M.J., SULON J., CARRO M.D., MANTECON A.R., BECKERS J.F., 1994. Plasmatic profiles of pregnancy-associated glycoprotein and progesterone levels during gestation in Churra and Merino sheep. Theriogenology, 42: 537-545.

20. RANILLA M.J., SULON J., MANTECON A.R., BECKERS J.F., CARRO M.D., 1997. Plasma pregnancy-associated glycoprotein and progesterone concentrations in pregnant Assaf ewes carrying single and twin lambs. Small. Rumin. Res., 24: 125-131.

21. RHIND S.M., GUNN R.G., DONEY J.M., 1983. A note on reproductive performance and plasma progesterone level during early pregnancy of Scottish Black Face and Cheviot ewes in relation to body condition and level of nutrition prior to mating. Anim. Prod., 37: 455-458. 
22. RONAYNE E., HYNES N., 1990. Measurement of plasma progesterone concentrations by extraction and non-extraction radioimmunoassays. Irish J. Agric. Res., 29: 109-115.

23. SAS, 1999. Statistical analysis system, Vers. 8. Cary, NC, USA, Statistic Institute.

24. SEMAMBO D.K.N., ECKERSALL P.D., SASSER R.G., AYLIFFE T.R., 1992. Pregnancy-specific protein B and progesterone in monitoring viability of the embryo in early pregnancy in the cow after experimental infection with Actynomyces pyogenes. Theriogenology, 37: 741-748.

25. SOUSA N.M., GARBAYO J.M., FIGUEIREDO J.R., SULON J., GONÇALVES P.B.D., BECKERS J.F., 1999. Pregnancy-associated glycoprotein and progesterone profiles during pregnancy and postpartum in native goats from the north-east of Brazil. Small Ruminant Res., 32: 137-147.

26. SZENCI O., HUMBLOT P., BECKERS J.F., SASSER R.G., SULON J. BALTUSEN R., VARGA J., BAJCSY C.A., TAVERNE M.A.M., 1999. Plasma profiles of progesterone and conceptus proteins in cows with spontaneous embryonic/foetal mortality as diagnosed by ultrasonography. Vet. J., 159: 287-290.

27. THIMONIER J., 2000. Détermination de l'état physiologique des femelles par analyse des niveaux de progestérone. Prod. Anim., 13 : 177183.

28. THORBURN G.D., SCHNEIDER W., 1972. The progesterone concentration in the plasma of the goat during the oestrus cycle and pregnancy. J. Endocrinol., 52: 23-36.

29. VANDAELE L., VERBERCKMOES S., EL AMIRI B., SULON J., DUCHATEAU L., VAN SOOM A., BECKERS J.F., DE KRUIF A., 2005. Use of a homologous radioimmunoassay (RIA) to evaluate the effect of maternal and foetal parameters on pregnancy-associated glycoprotein (PAG) concentrations in sheep. Theriogenology, 63: 1914-1924

30. VAN KAMPEN K.R., ELLIS L.C., 1972. Prolonged gestation in ewes ingesting Veratrum californicum: morphological changes and steroid biosynthesis in the endocrine organs of cyclopic lambs. J. Endocrinol., 52: $549-560$
31. VERBERCKMOES S., VANDAELE L., DE CAT S, EL AMIRI B., SULON J., DUCHATEAU L., DE KRUIF A., BECKERS J.F., VAN SOOM A., 2004. A new test for early pregnancy diagnosis in sheep: determination of ovine pregnancy associated glycoproteins (ovPAG) concentration by means of a homolgous radioimmunoassay. Flemish vet. J., 73: 119-127.

32. WALLACE J.M., AIKEN R.P., CHEYNE M.A., HUMBLOT P., 1997. Pregnancy-specific protein $B$ and progesterone concentrations in relation to nutritional regimen, placental mass and pregnancy outcome in growing adolescent ewes carrying singleton foetuses. J. Reprod. Fertil., 109: 53-58.

33. WILLARD J.M., WHITE D.R., WESSON C.A.R., STELFLUG J., SASSER R.G., 1995. Detection of fetal twins in sheep using radioimmunoassay for pregnancy-specific protein B. J. Anim. Sci., 73: 960-966.

34. ZARROUK A., ENGELAND I., SULON J., BECKERS J.F., 1999 Determination of pregnancy-associated glycoprotein concentrations in goats (Capra hircus) with unsuccessful pregnancies: a retrospective study. Theriogenology, 51: 1221-1231.

35. ZARROUK A., ENGELAND I.V., SULON J., BECKERS J.F., 1999 Pregnancy-associated glycoprotein levels in pregnant goats inoculated with Toxoplasma gondii or Listeria monocytogenes: a retrospective study. Theriogenology, 52: 1095-1104.

36. ZOLI A.P., BECKERS J.F. ECTORS F., 1990. Isolation of an ovine pregnancy specific protein. Theriogenology, 33: 366 (abstract).

37. ZOLI A.P., BECKERS J.F., WOUTERS-BALLMAN P., CLOSSET J., FALMAGNE P., ECTORS F., 1991. Purification and characterization of a bovine pregnancy-associated glycoprotein. Biol. Reprod., 45: 1-10.

38. ZOLI A.P., GUILBAULT L.A., DELAHAUT P., BENITEZ-ORTIZ W., BECKERS J.F., 1992. Radioimmunoassay of a bovine pregnancyassociated glycoprotein in serum: its application for pregnancy diagnosis. Biol. Reprod., 46: 83-92.

Reçu le 28.06.2006, accepté le 24.11.2006

\section{Summary}

Benyounes A., Lamrani F., Melo de Sousa N., Sulon J., Folch J., Beckers J.-F., Guellati M.A. Pregnancy Monitoring in Ouled Djellal Ewes by Pregnancy-Associated Glycoprotein and Progesterone Measurements

The aim of the present study was to characterize the plasmatic profiles of pregnancy-associated glycoproteins (PAG) and progesterone during gestation and two weeks postpartum in Ouled Djellal ewes. Twenty-five ewes were sampled weekly after estrus synchronization and natural mating. Concentrations of PAG and progesterone were determined by radioimmunoassay. Two ewes failed to become pregnant. Eighteen females developed normal ongoing pregnancies (12 single and 6 multiple) while 5 others were considered as having abnormal pregnancies. In normal ongoing pregnancies, PAG and progesterone concentrations were significantly influenced by the stage of pregnancy $(P<0.0001)$ but not by the fetal number. Individual plasmatic profiles were described in three ewes having had embryonic mortalities, in two having suffered fetal losses (at days 66 and 110), and finally in two ewes that had at the same time the presence of one alive and one or two mummified fetuses. Plasmatic concentrations of PAG were lower in ewes presenting simultaneously living and mummified fetuses.

Keywords: Sheep - Ouled Djellal ewe - Pregnancy Progesterone - Radioimmunoassay - Algeria.

\section{Resumen}

Benyounes A., Lamrani F., Melo de Sousa N., Sulon J., Folch J., Beckers J.-F., Guellati M.A. Seguimiento de la gravidez en la oveja Ouled Djellal mediante la dosificación de la proteína asociada a la gestación y de la progesterona

El objetivo del presente estudio fue la caracterización de los perfiles plasmáticos de la proteína asociada a la gestación (PAG) y de la progesterona durante la gravidez y dos semanas después del parto en la raza Ouled Djellal. Se tomaron muestras semanales de veinticinco ovejas después de la sincronización de los calores y la monta natural. Las concentraciones de PAG y de progesterona fueron determinadas mediante dosificación radio inmunológica. Dos hembras no fueron fecundadas, mientras que 23 tuvieron gestaciones consideradas normales $(n=18)$ o patológicas $(n=5)$. En 18 ovejas (12 gestaciones simples y 6 múltiples), las concentraciones de PAG y de progesterona fueron significativamente influenciadas por el estadio de la gestación $(\mathrm{P}<0,0001)$ y no por el número de fetos. Se describieron perfiles individuales en siete ovejas, de las cuales tres hembras con pérdidas embrionarias y dos ovejas con abortos (66avo y 110 avo días) y finalmente en dos ovejas que parieron a término, pero cada una un cordero vivo al mismo tiempo que expulsaron en el momento del parto uno o dos fetos momificados. Las concentraciones de PAG fueron más bajas en las hembras que tuvieron fetos momificados junto con fetos vivos.

Palabras clave: Ovino - Oveja Ouled Djellal - Gestación Progesterona - Técnica radioinmunologica - Argelia. 